\title{
Benjamin Jung, La bataille du placement. Organiser et contrôler le marché du travail, France, 1880-1918
}

Rennes, Presses universitaires de Rennes, coll. «Pour une histoire du travail », 2017

\section{Hugo Mulonnière}

\section{(2) OpenEdition}

\section{Journals}

Édition électronique

URL : http://journals.openedition.org/travailemploi/8075

DOI : $10.4000 /$ travailemploi.8075

ISSN : 1775-416X

Éditeur

DARES - Ministère du Travail

Édition imprimée

Date de publication : 1 janvier 2018

Pagination : 135-138

ISSN : 0224-4365

Référence électronique

Hugo Mulonnière, «Benjamin Jung, La bataille du placement. Organiser et contrôler le marché du travail, France, 1880-1918», Travail et Emploi [En ligne], 153 | janvier-mars 2018, mis en ligne le 11 juillet 2019, consulté le 25 septembre 2020. URL : http://journals.openedition.org/travailemploi/8075 ; DOI : https://doi.org/10.4000/travailemploi.8075 


\section{La bataille du placement. Organiser et contrôler le marché du travail, France, 1880-1918}

\section{Benjamin Jung}

Rennes, Presses universitaires de Rennes, coll. « Pour une histoire du travail », 2017, 296 p.

\section{Lu par Hugo Mulonnière*}

Avec cet ouvrage issu de sa thèse ${ }^{1}$, Benjamin Jung signe la première étude d'envergure spécifiquement consacrée au placement en France entre 1880 et la fin de la Première Guerre mondiale. Bien que sa constitution en objet de recherche historique soit ancienne ${ }^{2}$, le placement était jusqu'ici plutôt apparu comme un objet secondaire dans l'historiographie des politiques du travail et de l'emploi. B. Jung en fait son sujet central et analyse plus précisément la manière dont il est devenu un enjeu de mobilisations et d'interventions publiques sur la période.

Filant la métaphore tout au long du livre, l'historien s'attache à décrire la construction du placement comme enjeu politique et social sous l'aspect d'une « bataille » opposant des acteurs et des institutions présentés comme autant de belligérants. Son postulat est que ces concurrences, oppositions et compromis ont façonné le traitement de la question de l'accès à l'emploi et contribué à lui donner ses formes légales et institutionnelles. Parmi les acteurs qui s'affrontent, l'auteur identifie un certain nombre d' « intermédiaires du placement » (placeurs payants, organisations ouvrières, Bourses du travail, bureaux municipaux, sociétés philanthropiques), les pouvoirs publics, le Parlement, les réseaux réformateurs, ou encore le patronat, tous ayant des visées concurrentes, s'alliant ou s'opposant au gré des configurations, et participant à la co-production de l'enjeu collectif du placement. L'ouvrage de B. Jung consiste donc en la description et l'analyse de ce « champ de bataille » duquel naît l'action publique, avec pour objectif d'en saisir la pluralité des foyers et des formes.

\footnotetext{
* Groupe de recherche d'histoire (GRHis), université de Rouen ; Centre de recherche sociologique et politique de Paris/Cultures et sociétés urbaines (Cresppa/CSU).

1. Jung B. (2012), La Bataille du placement et les sans-travail. Concurrences entre intermédiaires et synthèse républicaine dans la genèse du marché du travail en France (1880-1914), thèse de doctorat d'histoire et civilisations, sous la direction d'André Gueslin, université Paris 7 Denis Diderot.

2. Voir par exemple Marquant R. (1962), « Les bureaux de placement en France sous l'Empire et la Restauration. Essais d'établissement d'un monopole », Revue d'histoire économique et sociale, vol. 40, n² 2, pp. 200-237 ; Luciani J. (1990), «Logiques du placement des ouvriers au XIX ${ }^{\mathrm{e}}$ siècle et construction du marché du travail », Sociétés contemporaines, $\mathrm{n}^{\circ}$ 3, pp. 5-18 ; de Larquier G. (2000), «Émergence des services publics de placement et marchés du travail français et britannique au Xx ${ }^{\mathrm{e}}$ siècle », Travail et Emploi, $\mathrm{n}^{\circ}$ 84, pp. 33-43.
} 
Une autre finalité du livre est de comprendre de quelle manière le marché du travail, dont l'organisation et le contrôle constituent un enjeu de la bataille du placement, a été construit. L'historien entend ainsi apporter sa contribution aux travaux qui, depuis plusieurs décennies, contestent sa «naturalité » ${ }^{3}$.

L'auteur commence tout d'abord par dresser une histoire sociale et spatiale des « stations d'embauchage » à la fin du XIX ${ }^{\mathrm{e}}$ siècle. La place de grève est un espace « proto-syndical » où se rencontrent offre et demande de travail, dans des conditions normées et contrôlées collectivement, mais cette organisation ne concerne que quelques professions dont le travail est le plus souvent recherché et qualifié. Le chapitre 2 est consacré aux bureaux de placement payants, « marchands de travail » qui concentrent les critiques et se retrouvent bientôt au cœur du débat public. Ils réalisent bien plus de placements que les intermédiaires gratuits, mais ne desservent qu'un très petit nombre de professions (domesticité, commerce et industries alimentaires). Au service du patronat, ils répondent aux impératifs de l'extrême flexibilité du travail des professions dans lesquelles ils sont présents (notamment celles de l'alimentation), tout en en tirant bénéfice. Après une description du monde des placeurs, B. Jung s'attache à caractériser les maigres efforts des autorités pour les encadrer et réprimer leurs abus. Ces deux premiers chapitres donnent ainsi à lire une histoire de la condition ouvrière à la fin du XIX ${ }^{\mathrm{e}}$ siècle par le prisme original du placement. On peut déplorer que cette démarche d'histoire sociale, «par le bas », ne soit pas davantage présente dans la suite de l'ouvrage. De même, une approche micro-historique centrée sur certains bureaux de placement, peut-être sous la forme d'une étude des guichets du placement, aurait été particulièrement féconde, notamment pour approfondir la caractérisation sociale de celles et ceux qui recourent aux placeurs.

Les deux chapitres suivants proposent une plongée dans des mouvements sociaux pour lesquels la question du placement a constitué un enjeu : le mouvement des sanstravail de 1883-1885 (chapitre 3), qui prend corps dans le secteur du bâtiment, et les campagnes menées par les syndicats de l'alimentation contre les placeurs commerciaux entre 1886 et 1893, cette fois-ci directement dans le but de les supprimer (chapitre 4). L'auteur s'attache à décrire le positionnement des diverses composantes du mouvement ouvrier (anarchistes et socialistes, eux-mêmes divisés en tendances rivales). Lors du mouvement des sans-travail, les organisations ouvrières réclament avant tout la protection de l'État, mais les déceptions face à l'attitude des Républicains au pouvoir, appelés « opportunistes » car ils acceptent d'atténuer leurs principes doctrinaux en fonction des configurations politiques, les conduisent bientôt à réorienter leurs revendications vers l'aménagement d'un lieu « dédié à l'accès contrôlé à l'emploi ». Celui-ci s'incarnera dans la création de la première Bourse du travail à Paris en 1887. Un autre grand moment de confrontation entre le mouvement syndical et l'État autour de la question du placement a lieu sous l'égide de la Fédération nationale des travailleurs de

3. Voir par exemple Torre-Schaub M. (2002), Essai sur la construction juridique de la catégorie de marché, Paris, LGDJ, 416 p. 
l'alimentation à partir de 1902 (chapitre 7). B. Jung met ainsi en lumière l'importance de l'accès au travail comme revendication du mouvement ouvrier - ce qui constitue d'ailleurs l'un des fils rouges de l'ouvrage - exposant la manière dont cette question a permis au champ syndical de se définir et de se structurer. L'ouvrage enrichit ainsi par son prisme original l'historiographie du syndicalisme et plus généralement du mouvement ouvrier.

Les cinquième et sixième chapitres sont centrés sur la question des Bourses du travail. La pensée de Fernand Pelloutier, pour qui les Bourses, notamment par leur fonction de placement, sont la base de la société future et la pierre angulaire de l'autoémancipation de la classe ouvrière, y est explicitée. Le président de la Fédération nationale des Bourses du travail en vient à envisager la rationalisation du placement via la création d'un organisme à l'échelle nationale. B. Jung montre comment, par le financement de cet organisme, le ministre du Commerce Alexandre Millerand (18991902) tente d'intégrer les syndicats au pacte républicain, suscitant des débats à la fois au sein de la classe politique et du mouvement ouvrier.

Dans le septième chapitre, sont retracées les dix années de la bataille qui mènent à la grande loi du 14 mars 1904 instaurant la gratuité universelle du placement. Ce cheminement vers la gratuité est traversé par une tension entre l'option d'un strict monopole syndical, rejetée par une majorité d'élus, et la solution consensuelle d'une prise en charge pluri-institutionnelle du placement. La loi de 1904 est finalement un compromis imparfait articulant l'extinction facultative du placement commercial, laissé à la charge des municipalités, et un encouragement timide des institutions de placement gratuit. Le chapitre suivant est consacré aux conséquences de la mise en application de la loi et à l'attitude des différents acteurs face à cette nouvelle configuration. La CGT, au faîte de sa période syndicaliste-révolutionnaire, favorise les mots d'ordres de lutte : la renonciation à la revendication du monopole syndical sur le placement se profile. L'auteur discute ensuite l'hypothèse selon laquelle l'application de la loi de 1904 aurait mis en concurrence les Bourses du travail et les bureaux municipaux ${ }^{4}$. Il insiste quant à lui sur leur complémentarité, en montrant que les municipalités ont eu tendance à s'appuyer sur elles, choisissant notamment de ne pas installer d'office municipal lorsqu'une Bourse préexistait. Cependant, ce choix a renforcé le contrôle des autorités locales sur leur fonctionnement, les syndicats dépendant de leur bonne entente avec ces dernières. Les relations entre syndicats et autorités locales ne tardent d'ailleurs pas à se détériorer, contribuant à une marginalisation des Bourses dont l'auteur n'aura de cesse de souligner les symptômes jusqu'à la fin de la période.

Les deux derniers chapitres de l'ouvrage, consacrés aux années 1910-1918, restituent le deuxième grand moment de la bataille, durant lequel le jeune ministère du Travail occupe un rôle central, engageant les prémices d'une action publique en matière de placement et œuvrant pour le développement du paritarisme. Ce mouvement est concrétisé par le décret du 25 octobre 1911 qui incite notamment les municipalités à

4. Luciani J. (1990), « Logiques du placement des ouvriers au XIX ${ }^{\mathrm{e}}$ siècle... », art. cit. 
nommer des commissions paritaires. Le ministère est alors fortement influencé par les milieux réformateurs, partageant leur volonté d'organisation du marché du travail et de pacification des relations sociales. Sous leur influence, la question du placement est désormais perçue par les autorités comme un levier de résorption du chômage. Ces projets qui concurrencent directement les services syndicaux et les Bourses du travail ne font pas l'unanimité dans les organisations ouvrières, mais certains syndicats commencent à s'y rallier à la veille de la Première Guerre mondiale. La Grande Guerre accélère le processus à travers l'établissement par les pouvoirs publics d'un système de placement pensé comme un outil stratégique au service de l'économie de guerre. Le rôle clé y est détenu par les Offices départementaux de placement, dont la plupart fonctionnent avec une commission administrative paritaire, coordonnés entre eux et en rapport permanent avec un Office central de placement.

En somme, l'angle d'observation et la méthodologie adoptés par l'auteur, fondés essentiellement sur l'analyse des interactions des forces antagonistes en présence, apparaissent très féconds. On peut cependant regretter que l'ouvrage délaisse quelque peu l'analyse des réseaux, des circulations d'idées et de savoirs ainsi que des processus décisionnels au sein de chacune des forces en présence. En effet, esquissée dans le cas des organisations du mouvement ouvrier, la démarche est brossée trop rapidement pour l'État, les réformateurs, les placeurs ou encore le patronat, qui constituent pourtant des catégories d'acteurs hétérogènes et non univoques. Cela ne saurait amoindrir le fait que, par son analyse fine et rigoureuse des configurations de la « bataille du placement », le livre de B. Jung est d'un grand intérêt pour qui s'intéresse à la construction des mobilisations et des politiques publiques de l'emploi. 